\title{
Predicting Performance of Collaborative Storytelling Using Multimodal Analysis
}

\author{
Shogo OKADA ${ }^{\dagger a}$, Member, Mi HANG ${ }^{\dagger}$, Nonmember, and Katsumi NITTA $^{\dagger}$, Member $^{2}$
}

\begin{abstract}
SUMMARY This study focuses on modeling the storytelling performance of the participants in a group conversation. Storytelling performance is one of the fundamental communication techniques for providing information and entertainment effectively to a listener. We present a multimodal analysis of the storytelling performance in a group conversation, as evaluated by external observers. A new multimodal data corpus is collected through this group storytelling task, which includes the participants' performance scores. We extract multimodal (verbal and nonverbal) features regarding storytellers and listeners from a manual description of spoken dialog and from various nonverbal patterns, including each participant's speaking turn, utterance prosody, head gesture, hand gesture, and head direction. We also extract multimodal co-occurrence features, such as head gestures, and interaction features, such as storyteller utterance overlapped with listener's backchannel. In the experiment, we modeled the relationship between the performance indices and the multimodal features using machine-learning techniques. Experimental results show that the highest accuracy $\left(R^{2}\right)$ is 0.299 for the total storytelling performance (sum of indices scores) obtained with a combination of verbal and nonverbal features in a regression task.
\end{abstract}

key words: storytelling performance, multimodal interaction, inference, data mining, small group, conversation analysis

\section{Introduction}

Storytelling involves the conveying of events to a listener using words, sounds, and actions, in order to reveal the image of a story. Storytelling is both a kind of explanation and a method for explanation. Good storytelling not only helps the listener to understand the story, but also makes conversation entertaining. Therefore, it is an important method of sharing information, presenting, teaching, etc. In recent years, the importance of storytelling techniques has also been recognized in business [1], because it is the first step in stating ones' own opinion in a logical manner to others. The military, aviation, medical, and law communities are groups which rely heavily on storytelling as a method for teaching the key principles of their discipline [2].

In storytelling, adequate performance is required to communicate the content of the story to the listener correctly and to make the listener imagine the story. Persuasiveness, which is a key aspect of storytelling performance, is controlled by both verbal communication and nonverbal behavior during storytelling [3], [4]. Previous analysis suggests that the nonverbal behavior of the storyteller can be used

\footnotetext{
Manuscript received September 30, 2015.

Manuscript revised March 15, 2016.

Manuscript publicized April 1, 2016.

†The authors are with Tokyo Institute of Technology, Yokohama-shi, 226-8503 Japan.

a)E-mail: okada@c.titech.ac.jp

DOI: 10.1587/transinf.2015CBP0003
}

to improve storytelling performance and enhancing persuasiveness. Although the importance of storytelling is recognized by researchers [1], [2], there is very limited work conducted on modeling storytelling performance from a computational approach.

From this background, we focus on computational modeling to predict storytelling performance in a group conversation based on verbal and nonverbal information. When more than two participants (storytellers) in a group know a story, storytelling can become more entertaining by integrating different aspects of a story from multiple participants. Each participant can complement the content of a story told by another participant, when there is lack of information.

The main purpose of computational modeling in this study is as follows. The computational model has several potential applications such as a system to evaluate storytelling performance and a system to retrieve effective or attractive storytelling from large amounts of storytelling conversation dataset. The retrieved storytelling is available as a reference or teaching material for users who want to tell or teach the story (content). Therefore, the modeling conducted in this study is a first important step toward the implementation of these applications.

Through developing the computational model, we can investigate the relationship between the performance of storytellers and multimodal (verbal and nonverbal) features extracted from participants in a conversation. This investigation reveals the kind of verbal and nonverbal features observed from the group with high storytelling performance. We set three hypotheses to extract various kinds of features to improve the prediction accuracy of storytelling performance in a group conversation.

[Hypothesis 1 (H1):] The results of [5] showed that the prosodic features extracted from the voice of the speaker are important for predicting the quality of the explanation. This finding is also available in predicting storytelling performance. Gestures allow the sending of information smoothly in a storytelling task [6]. Gazing at the listener is an important behavior, as it makes the listener focus attention on the storytelling [7]. Furthermore, [8] showed that utterances (verbal information) by a speaker could be used to effectively predict the persuasiveness of the speaker. From the findings in these previous studies, both verbal and nonverbal information, including audio and visual cues, are important to predict the performance. 
[Hypothesis 2 (H2):] Response behavior including backchannel and nodding observed by the listeners can sometimes show whether they understand the story. In fact, the relationship is collaborative, and the narrator extracts responses from the listeners in a narrative interaction. Therefore, it is important to obtain not only multimodal features from the storyteller, but also from the listeners [9].

[Hypothesis 3 (H3):] Conversation is a dynamic phenomenon observed as an interaction among all participants. Thus, capturing the interaction pattern helps us to understand the relationship between these participants. For example, patterns such as "storyteller speaks to listener using hand gesture" and "a listener and a storyteller nod toward another storyteller" display the relationship. These patterns are effective features to predict the performance.

Based on these hypotheses, we propose a multimodal modeling framework to develop a computational model that predicts storytelling performance and to evaluate the model in this study. We also analyze the relationship between spoken word features extracted from manual transcription, nonverbal behaviors, and the performance, in small groups. We first present related work in Sect. 2. In Sect. 3, we present an overview of modeling and data collection for analyzing the performance. Section 4 explains our multimodal pattern annotation scheme. We explain the extracted multimodal features in Section Sect. 5. We also perform correlation analysis and regression prediction task in Sect. 6 and discuss the effective features to improve the accuracy in Sect. 7. Finally, we discuss for future works in Sect. 8 and present our conclusions in Sect. 9.

\section{Related Work}

\section{Storytelling and nonverbal behavior}

There has been research in communication science and sociolinguistics analyzing the relationship between nonverbal behavior and storytelling or explanation. [4] suggests that it is important to analyze nonverbal behavior to understand speaker persuasiveness. This work analyzes the relationship among nonverbal behaviors, dimensions of source credibility, and persuasiveness in explanation of videotaped speeches by 60 speakers. It reports that persuasiveness is correlated with vocal pleasantness, kinesic and proxemic immediacy, facial expressiveness, and kinesic relaxation.

[3] analyzes multimodal nonverbal patterns of participants in a storytelling conversation task conducted to learn a second language. It is noted that learners (participants) engage in cooperative talk and learning using multimodal nonverbal behaviors such as hand gestures. Such research shows that nonverbal behavior plays an important role in storytelling. However, it does not focus on building computational models to infer the performance of storytelling. We focus on modeling to infer performance via audio, signal processing, pattern recognition, and machine learning.

\section{Computational multimodal analysis for group meetings}

There have been many studies on nonverbal behavior analysis in small groups with pattern recognition techniques. On the one type of these researches, multi modal sensing system are developed to capture conversation phenomena such as who is speaking, who is looking at whom using speech, image processing and machine learning [10].

Other type of the research focus on inferring people's traits, personality, or internal states. In [11] an analysis of personality prediction was conducted, using self-reported questionnaires from the participants themselves. In [12], an analysis of personality prediction in small groups was conducted on the basis of trait attributes from external observers. There have been studies on role recognition in groups. This research is split into recognition tasks of formal [13] or informal roles [14], [15]. In [16], a multimodal analysis is conducted for inferring emergent leadership in small groups. [17] proposed the framework to detect interest level in meeting, which is annotated by external observers from audio-visual data. These studies do not focus on multimodal analysis for inferring storytelling performance. We collect a multimodal data corpus including indices of performance annotated by external observers for modeling storytelling performance.

\section{Computational modeling for storytelling performance}

Persuasiveness is an important aspect of performance in storytelling. [8] proposed a multimodal approach for predicting a speaker's persuasiveness in online social multimedia content. [18] analyzes the persuasion of candidates in job interviews (dyadic interaction setting) using a computational multimodal approach. In these studies, speech audio features, speech turns, and body motion are effective features for predicting persuasiveness. Their research target such as social media and dyadic interaction, is different from that of our research.

There are existing researches which focus on modeling to evaluate speaking performance. [5] analyze acousticprosodic features correlated with a good speaker. [19] analyze to identify both audio and visual nonverbal behaviors contribute public speaking performance evaluated by an expert annotator using machine learning techniques. In this research, prosodic features, arm and leg motion, and face direction are extracted automatically from speaker using a microphone and a depth camera. [19] shows face direction and motion are important to be assessed as a good speaker. Though their researches do not consider with listener's behavior (nodding and back-channel) and interaction between speaker and listener, these studies support our hypothesis that visual-audio nonverbal cues are effective features for predicting storytelling performance.

\section{Interaction feature extraction in conversation}

In the proposed multimodal approaches to analyzing a conversational dataset, we extracted co-occurrence multimodal features and interaction features between storyteller and listener using pattern discovery as a data mining ap- 


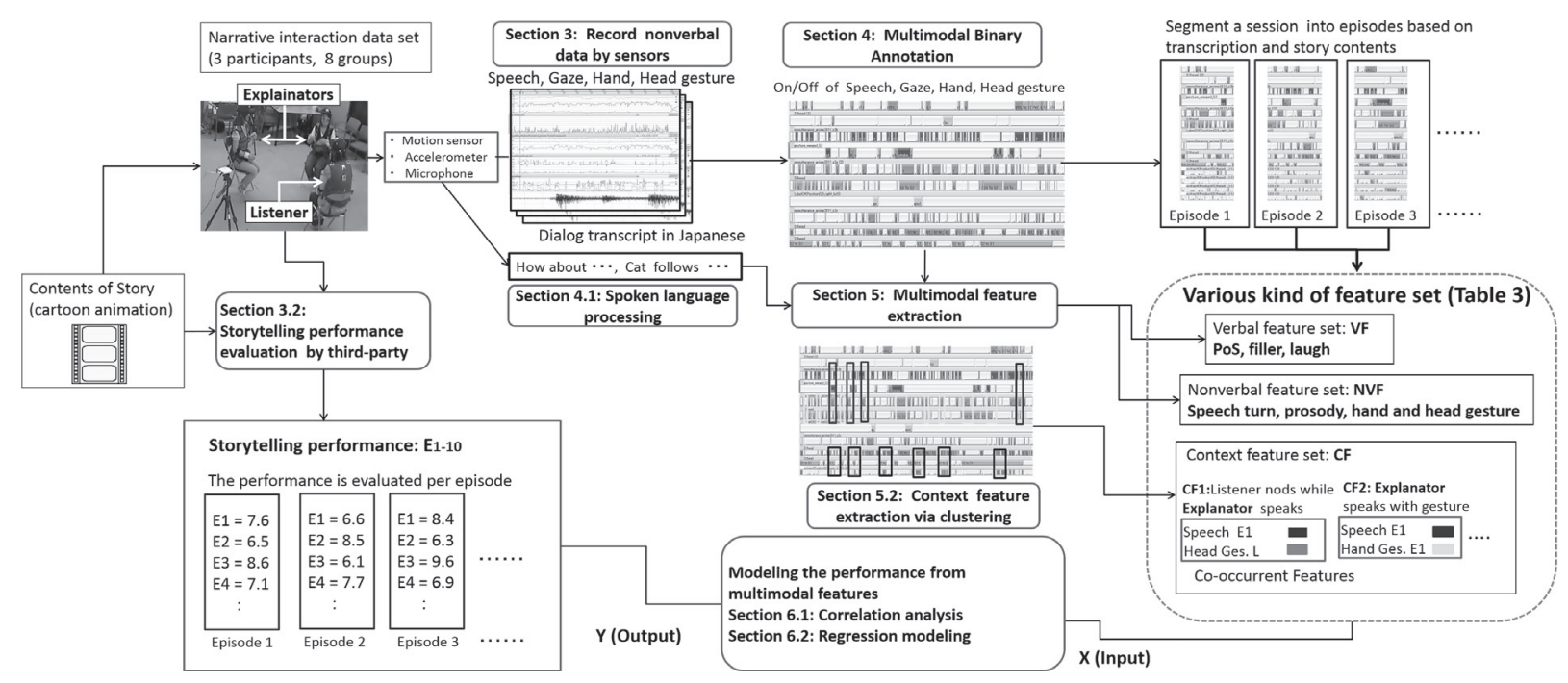

Fig. 1 Overview of multimodal analysis for storytelling performance

proach. [20] shows that using contextual cues (prosodic and lexical features) related to the speaker improves the recognition accuracy of the listener's head nods in a dyadic interaction setting. That research focuses on a listener's head gesture recognition in a dyadic interaction. [21], [22] use a latent Dirichlet allocation (LDA) model for mining context features in group. In [22], group features for speaking status and gaze state are used for input to an LDA. Context features are extracted as LDA topics (clusters). Conversely, we used the various kind of feature set not only speech and gaze cues, but also hand and head gesture features for modeling the performance. Main objective of [21], [22] is finding context features to link role of participant or personality trait. Our objective is modeling to predict storyteliing performance using context features and is quite different with these objective.

\section{Contributions of this research}

The results of the present study contribute to this research field in two ways. First, computational multimodal analysis and modeling to predict storytelling performance in a small group is conducted as a novel problem. We collect a new data corpus for this study. Second, we extracted various types of multimodal feature sets, including verbal features, low-level nonverbal features, co-occurrence nonverbal features, and interaction features to predict performance.

\section{Overview of Storytelling Performance Modeling}

Figure 1 shows an overview of the multimodal analysis for storytelling performance in this research. First, we collect a new multimodal data corpus by enhancing the data corpus collected in [23]. This corpus includes (1) manual transcription data of spoken dialogs; (2) various nonverbal features observed by the participants: speech, hand gestures, head gestures, and head direction to approximate gaze direction; and (3) multiple indices of the storytelling performance annotated by external observers. In a conversation task, two storytellers collaboratively tell a story to a listener who does not know the content of the story. To measure various aspects of the storyteller's performance, we set ten performance indices, including the precision of the story told, storytelling enthusiasm, speaking fluency, speaking liveliness, etc.

Second, we extract various types of nonverbal features. Low-level features are extracted to test $\mathrm{H} 1$, including speech length, speech turn, gaze direction, head gestures, and hand gestures. These features are extracted from both storytellers and from the listener $(\mathrm{H} 2)$. We also extract multimodal cooccurrence features, such as utterance with head or hand gestures, and interaction features, such as mutual gaze and utterance of storyteller overlapped with listener's backchannel (H3). We define these features as "Context features" because these features capture conversational contexts such as more detail in conversation or nonverbal behavior than the low-level features. In our experiment, we train the model to predict the performance based on a multimodal feature set using a regression model. We also analyze effective combinations of features to predict performance.

\subsection{Storytelling Interaction Dataset}

Toward modeling storytelling performance, we prepare a specific conversational setting as follows. Participants in a group are asked to perform only storytelling during the conversation. In addition, the role of each participant (storyteller or listener) is fixed in this setting. In many casual conversations (e.g., the task used in [10]), these roles are not assigned to participants, and the content of the conversation is not restricted, as it is in our setting. This point is the main difference between the other conversation corpora and our corpus, which is specified for modeling storytelling performance.

In our task, two participants narrate a cartoon story to a participant from memory. The name of the cartoon story is "Canary Row," a story that has been used for gesture analy- 
sis in narrative tasks [24]. The setting of a dyadic narrative interaction designed by McNeill [24] is changed to that of a small group narrative interaction. In this setting, a group is composed of three participants, two of whom have watched the video, and tell it to the third. The three participants were not previously acquainted with each other and have never watched the video or listened to the story. Storytellers are instructed to enable the listener to understand the details of the story. To promote understanding, the listener can ask questions freely and the two storytellers can also discuss the story.

According to [23], 24 women participants aged 20 to 25 years are recruited through a temporary employee company. An eight-group conversation dataset is collected in cooperation with these participants. The average length of the recorded datasets is $11 \mathrm{~min}$, and the total is $700 \mathrm{~min}$. The story comprises eight short episodes. Storytellers in all sessions were required to explain for an entire session. Some storytellers forgot telling episodes. All storytellers began to explain each episode after telling the prologue (i.e., main characters and overview of a story). In total, group conversation data included a maximum of nine episodes (prologue + eight). A group interaction sequence dataset is segmented manually into the subsequence datasets with nine episodes. We collected a total of 67 subsequences (episodes) as data from an eight-group conversation dataset.

\subsection{Questionnaire Variables Measuring Performance}

Our goal in this research is to predict well-organized and attractive storytelling that can be a reference of external observers. The performance of storytelling was evaluated by questioning external observers, who also had never watched the video or listened to the story. Third-party participants (Evaluators) watched both the contents of the video "Canary Row," the subject of the storytelling, and the video that captures group conversations and storytelling. They evaluated which group could tell the video contents as a story faithfully by comparing the story and video contents. We prepared ten statements in Table 1 to evaluate various aspects of storytelling. $E_{1-10}$ capture performance of "Eloquence", "Enthusiasm", "Fluentness", "Wittiness", "Preciseness", "Compactness", "Summarization", "Liveliness", "Cooperation", "Adequately answering to questions $(Q \& A)$ ", for storytellers, respectively. The ten variables were evaluated on a ten-point scale. Three evaluators scored all of the episode data (67 episodes). Figure 2 shows the distribution of scores marked by the evaluators using a boxand-whisker plot. It reports that variance of scores by Evaluator 1 is largest.

The agreement between the annotators for each performance index is given in Fig. 3, in terms of the intracorrelation coefficient $(\operatorname{ICC}(1,1), \operatorname{ICC}(1, \mathrm{k}))$ and Cornbach's alpha $(\alpha)$ measures. All of the agreement values are sufficient for all indices, because all values of $\operatorname{ICC}(1, k)$ are greater than 0.7. By comparing story telling to the original video, annotators could evaluate the quality of the storytelling. As
Table 1 Statements for evaluating storytelling performance

\begin{tabular}{|l|l|l|}
\hline Index & Abb. & Questionnaire \\
\hline$E_{1}:$ (Eloquence $)$ & Eloquent. & Do they speak eloquently? \\
$E_{2}:$ (Enthusiasm $)$ & Enthus. & Do they have enthusiasm for storytelling? \\
$E_{3}:$ Fluentness $)$ & Fluent. & Do they speak fluently? \\
$E_{4}:$ (Wittiness $)$ & Witt. & Do they explain wittily? \\
$E_{5}:$ (Preciseness $)$ & Precise. & Do they provide precise information? \\
$E_{6}:$ (Compactness $)$ & Compact. & Do they explain compactly? \\
$E_{7}:($ Summarization $)$ & Summary & Do they emphasize the main point? \\
$E_{8}:$ (Liveliness $)$ & Live & Do they become lively? \\
$E_{9}:($ Cooperation $)$ & Cooperate. & Are they cooperative with each other? \\
$E_{10}:($ Q\&A $)$ & Q\&A & Can they answer listener's questions? \\
\hline \hline$E_{\text {Total }}$ & Total & Sum of $E_{1}-E_{10}$ \\
\hline
\end{tabular}

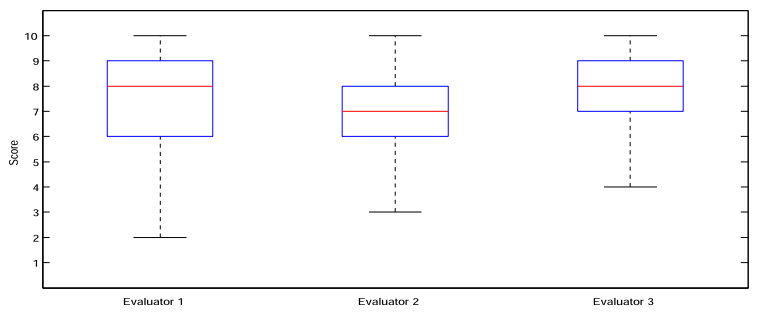

Fig. 2 Distribution of scores marked by the annotators on the performance indices

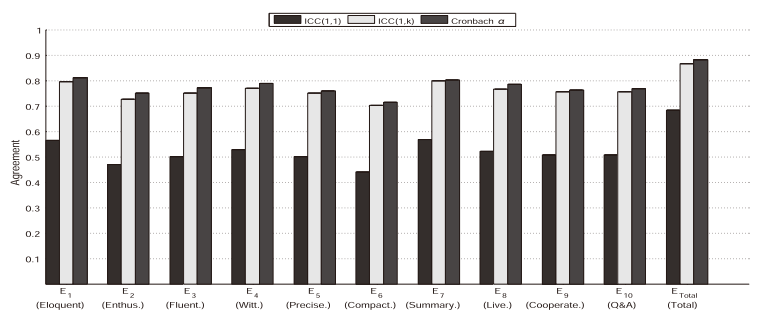

Fig. 3 Agreement between the annotators on the performance indices

a result of the annotations, higher agreement measures are obtained. We use the average value of scores by three evaluators for target variables as the quality of storytelling.

\section{Multimodal Behavior Annotation}

[23] discusses autonomous annotations derived from lowpower sensor data on a multimodal dataset. Head and hand gestures are sensed using a wearable motion capture system and accelerometer sensors. Wireless microphones (head-set type) are used to collect voice data of participants. We use the binary annotation data of nonverbal behaviors: speech status, hand gestures, head gestures, and head direction shared by authors of [23].

\subsection{Spoken Language Annotation}

Vocabulary and grammar are important features for evaluating storytelling performance, because storytelling with wide vocabulary (noun and verb) help listeners understand video contents. We extract features from spoken language data to capture the vocabulary of participants. In particular, we count number of words spoken per type of grammatical construction. Manual transcripts from spoken language are created by two expert coders. Word features are extracted from transcriptions using a Japanese morphological analy- 
sis tool: Chasen ${ }^{\dagger}$, because all participants are Japanese and speak Japanese. First, the sentence is segmented into word set in the process of the tool by tokenization of sentences in manual transcription of spoken dialog. Second, type of part of speech (PoS) is annotated automatically to each word in the word set. We count words $w_{1-10}$ spoken for each grammatical construction or PoS. $w_{1-10}$ denotes number (count) of "noun", "conjunction", "verb", "adjective", "adverb", "particle", "auxiliary verb", "interjection and backchannel", "filler", and "laugh".

Filler and laugh labels are also annotated to each statement by the coders. The amount of filler influences the fluentness of speech, and laughter is a useful cue for detecting conversational signals. For example, agreement and jokes can be identified with the help of laugh detection [25]. Interjection and backchannel also are important features to capture behavior of listener. Extracted features of grammatical constructions are as follows.

\subsection{Nonverbal Behavior Annotation}

Nonverbal behavior is annotated into two label of $O n / O f f$ by binary segmentation of continuous signal data observed from sensors. We extracted nonverbal features from segments where the label is $O n$ after binary annotation. We briefly introduce the procedure for nonverbal behavior annotation. Details of the procedure are described in [23].

\section{Speech status annotation:}

We use standard speech signal processing techniques to automatically detect speaking status as a binary variable to indicate whether a participant is speaking. Julius software is used for this implementation ${ }^{\dagger \dagger}$. We define the speech segment $(s p)$ set labeled to $O n$ as speech status dataset $S P$. Each segment $s p$ has a time length.

\section{Hand gesture status annotation:}

Hand gesture status is annotated manually as a binary variable indicating list motion of participants or nonmotion by three annotators. We define the hand gesture segment $(h g)$ set labeled to $O n$ as hand gesture status dataset $H G$.

\section{Head gesture status annotation:}

Head gesture status is also annotated manually as a binary variable indicating vertical motion or nonmotion by one annotator. We define the head gesture segment (he) set labeled to $O n$ as head gesture status dataset $H E$.

\section{Gaze direction annotation:}

To detect gaze direction, head pose and head direction were used for the approximation of gaze direction. Head direction patterns were manually annotated as binary variables indicating whether a participant is facing the right participant or left participant. Therefore gaze direction status $G_{i j}$ is annotated in where participant $i$ looks at $j$. We define the gaze direction status $g_{i j}$ labeled to $O n$ as gaze direction status dataset $G_{i j}$.

[23] reports the binary annotation of hand gesture

\footnotetext{
${ }^{\dagger}$ Chasen: http://chasen.naist.jp/hiki/ChaSen/

$\dagger$ Julius: http://julius.sourceforge.jp
}

(on/off), head gesture (on/off) and head direction (right/left) annotation were moderately successful. Using machine learning techniques (HMM, KNN, and Linear SVM) and , F value of test data is $0.89,0.71$, and 0.84 , respectively. In this study, we use manual-annotation labels in feature extraction and preprocessing for gesture and gaze direction in following section, because the objective is to evaluate whether storytelling performance can be predicted from combinations of verbal and nonverbal information observed from participants.

\section{Multimodal Feature Extraction}

We extracted various multimodal features linking storytelling performance from multiple label segments annotated in [23] ( $S P, H G, H E, G)$ and verbal features set in this re$\operatorname{search}(W)$.

\subsection{Feature Description from Each Modality}

In this task setting, the roles of the three participants in a group are assigned to storyteller or listener. We extract features as per each role from verbal and nonverbal label segments. For storytellers, we calculate the features based on sum of value of the features extracted from both storytellers. The main reason is the fact that we need to compare feature vectors observed from each group, because performance score is assigned not to each storyteller separately, but to each episode spoken in the group. Let us assign the participants the IDs $E 1, E 2, L$, where $E 1, E 2$ are the IDs of the storytellers and $L$ is the ID of the listener.

\subsubsection{Procedures of Feature Extraction}

We extracted features from the nonverbal segments and spoken words observed between the start frame $s t$ and the end frame $e t$ of each episode $S E$ in the interaction data. Note that $S P, H G, H E$, and $G$ are label segment dataset where each segment has own time length, and $W$ is composed of the count of words $w$ spoken per type of grammatical construction. value of all variables defined in the following section is divided by the length of each episode and normalized.

\subsubsection{Spoken Word Features}

Grammatical construction (Sect. 4.1) $W_{i, j}$ is calculated for each participant $i(i=\{E 1, E 2, L\})$ from spoken words observed in $j$ th episode $S E_{j}$. Features of storytellers are defined as the total of the grammatical construction frequency used by two storytellers $E W_{j}=W_{E 1, j}+W_{E 2, j}$ and features of listener are defined as $L W_{j}=W_{L, j}$. The spoken word feature set is $W F_{j}=\left\{E W_{j}, L W_{j}\right\}$, a 20-dimensional vector because $W_{j}$ is ten dimensional vector.

\subsubsection{Speech Features}

Let the speech segment set of the participant $i$ be $S P_{i}$ observed in an episode $S E$, which has segments $s p_{i, k}, \quad(1 \leq$ 
$k \leq N_{i}$ ), where $N_{i}$ denotes count of segments in $S P_{i}$. Speech length and Utterance count:

Total speech length $S L_{i}$, speaking turn (utterance count) $S T_{i}$ of participant $i$, total length of storytellers $E S L$, and total time of storytellers EST are calculated as follows.

$$
\begin{array}{r}
S L_{i}=\sum_{k}^{N_{i}} S L_{i, k}, \quad S T_{i}=N_{i} \\
E S L=S L_{E 1}+S L_{E 2}, \quad E S T=S T_{E 1}+S T_{E 2}
\end{array}
$$

where, $S L_{i, k}$ denotes the length of $s p_{i, k}$, and the length and time of the listener are $L S L=S L_{L}, L S T=S T_{L}$, respectively.

\section{Turn taking count:}

Count of turn taking is defined as the time $T T$ that floor is moved from a participant $i$ to other participant $i^{*}\left(i \neq i^{*}\right)$. We count when $s p_{i, k}$ starts after $s p_{i^{*}, k^{*}}$ and $s p_{i, k}$ ends after $s p_{i^{*}, k^{*}}$ as one turn.

\section{Energy and Pitch:}

Prosodic features are also important to capture the characteristics of storytellers. According to [18], the energy of the voice is effective in predicting the persuasiveness of a speaker in an interview. Prosodic feature have important roles in impressive storytelling. Based on the binary speech segment, we obtained the speech signal for each participant. We extracted two prosodic speech features, energy and pitch, computed for the signal extracted for each individual participant. Following the same procedure with extraction of speech length, we extracted maximum, minimum, mean, and standard deviation of energy in each speech segment for storytellers as and extracted these features of pitch. In total, the speech feature set is a 13-dimensional vector. We used the speech feature extraction $\operatorname{code}^{\dagger}$ to extract the prosodic features.

\subsubsection{Gaze Features}

Consider the gaze segment set $G_{i, l}(i \neq l)$ observed in an episode $S E$, where each segment is $g_{i, l, k}, 1 \leq k \leq N_{i, l} . g_{i, l, k}$ indicates that participant $i$ looks at $l$.

Time length of gaze segment: Time length, where participant $i$ looks at $l$, is defined as $G L_{i, l, k}$, the length of segment $g_{i, l, k}$. Time length $E G F$ focusing on storytellers are defined as $G F_{i}=\sum_{l, k} G L_{i, l, k}, E G F=G F_{E 1}+G F_{E 2}$. Listener's time length is $L G F=G F_{L}$.

\section{Time length of mutual gaze:}

Let mutual gaze feature be the number of frames $M G_{i, l}$ during which two participants $i, l$ look at each other. Time length of a mutual gaze between two storytellers is $E M G=$ $M G_{E 1, E 2}$, and time length of a mutual gaze between an storyteller and a listener is $L M G=\left(M G_{E 1, L}+M G_{E 2, L}\right) / 2$.

\section{Count that gaze direction changes:}

We measure the time $G T_{i, l}$ that the gaze direction of participant $i$ is changed during an episode $S E$. The calculation method is the same as that of $T T$. The feature of storytellers

\footnotetext{
${ }^{\dagger}$ Speech feature extraction code, http://groupmedia.mit.edu/ data.php.
}

is $E G T=\left(G T_{E 1}+G T_{E 2}\right) / 2$, and the count that gaze direction of listener changes is $L G T=G T_{L}$. In total, the gaze feature set is a six-dimensional vector.

\subsubsection{Head and Hand Gesture Features}

Let $H E_{i}$ and $H G_{i}$ be the sets of head and hand gesture segments, respectively, observed in $S E$. We calculate the number of segments and segment lengths in the same manner. The total head and hand gesture lengths $H L_{i}, G L_{i}$, the gesture times $H T_{i}, G T_{i}$ of participant $i$, the total length of storytellers $E H L, E G L$, and the total time of storytellers $E H T, E G T$ are calculated as follows. In total, the hand gesture and head gesture feature set are $H G F=\{E G E L$, $L G E L, E G E T, L G E T\}$ and $H E F=\{E H L, L H L, E H T$, $L H T\}$, respectively, which are four-dimensional vectors. We summarize the extracted multimodal features set in Table 2.

\subsection{Context Feature Extraction}

Conversational context features are extracted, as defined as multimodal co-occurring patterns (e.g. storyteller's speech segments $S P_{E 1}$ is co-occurring with the head gesture $H E_{L}$ of the listener). For example, simultaneously with the listener's nodding, we sometimes observe that a storyteller speaks to the listener while looking at him/her. In this case, the storyteller' s speech segments $S P_{E 1}$ and gaze segments $G_{E 1, L}, G_{L, E 1}$ are co-occurring with the head gesture $H E_{L}$ of the listener. These multi modal co-occurring patterns some-

\begin{tabular}{|c|c|c|}
\hline & Variables & Feature description \\
\hline & $E P L$ & Time length of each episode \\
\hline \multirow{2}{*}{$>$} & \multicolumn{2}{|r|}{ Word Features } \\
\hline & $E W_{1-10}$ & Count of words in utterance by storytellers \\
\hline & \multicolumn{2}{|r|}{ Speech features } \\
\hline \multirow{24}{*}{$\begin{array}{l}\frac{1}{z} \\
z\end{array}$} & $E S L$ & Time length of utterance by storytellers \\
\hline & $L S L$ & Time length of utterance by listener \\
\hline & EST & Count of utterance by storytellers \\
\hline & $L S T$ & Count of utterance by listener \\
\hline & $T T$ & Count of turn taking \\
\hline & ESE & Energy features of storytellers (min, max, mean, var) \\
\hline & ESP & Pitch features of storytellers (min, max, mean, var) \\
\hline & \multicolumn{2}{|r|}{ Gaze features } \\
\hline & $E G F$ & Time length while storytellers are focused \\
\hline & $L G F$ & Time length while listener is focused \\
\hline & $E M G$ & Time length of mutual gaze: Exp-Exp \\
\hline & $L M G$ & Time length of mutual gaze: Exp-Lis \\
\hline & EGT & Count of change of gaze state: Exp \\
\hline & $L G T$ & Count of change of gaze state: Lis \\
\hline & \multicolumn{2}{|r|}{ Hand gesture features } \\
\hline & EGEL & Time length of gesture segment by storytellers \\
\hline & LGEL & Time length of gesture segment by listener \\
\hline & EGET & Count of gesture segment by storytellers \\
\hline & LGET & Count of gesture segment by storytellers \\
\hline & \multicolumn{2}{|r|}{ Head gesture features } \\
\hline & EHL & Time length of head gesture by storytellers \\
\hline & LHL & Time length of head gesture by listener \\
\hline & EHT & Count of head gesture by storytellers \\
\hline & LHT & Count of head gesture by listener \\
\hline \multirow[b]{2}{*}{ 已゙ } & \multicolumn{2}{|r|}{ Context (multimodal nonverbal) features } \\
\hline & $C E_{1-195}$ & Count which pattern is observed \\
\hline
\end{tabular}

Table 2 Extracted multimodal features 
times capture the characteristics of conversational scenes better than single modal patterns. We modify the algorithm proposed in [26] to efficiently extract the co-occurring patterns from continuous time-series data. We modify the algorithm at minor points for this purpose. The proposed algorithm is as follows.

Input: Nonverbal segment sets defined in Sect. 4.2: $S P, G, H E, H G$,

Output: Context pattern set: $C F$

Step 1: Construct a coincidence graph $G$ as a directed graph $G=(V, E)$. Vertex $V$ is a nonverbal segment, and $E$ is the weight calculated based on the coincidence frequency between the segments.

Calculation of weight $E$ : Each segment $N F_{o}$ $(S P, G, H E, H G)$ is represented by a vertex $v_{o} \in V$. Weight $w_{o, p}$ of edge $e_{o, p}$ (edge connecting vertex o to vertex $\mathrm{p}$ ) is calculated as: $e_{o, p}=$ overlap $\left(N F_{o}, N F_{p}\right) / N_{o}$, where overlap $\left(N F_{o}, N F_{p}\right)$ is the total number of overlapping occurrences between occurrences of $N F_{o}$ and $N F_{p}$. For example, $\operatorname{overlap}\left(S P_{E 1}, H E_{L}\right)=50$, where 50 of total segments in the speaking segments of a storyteller $S P$ overlap temporally with the head gesture segments $H E$. $N_{o}$ is the number of segments in $N F_{o}$. Here, we defined two label segments have temporal overlap if more than one frame is overlapped in the two segments.

Step 2: Graph clustering is preformed to find the multimodal context clusters $C E$ using Algorithm 1. $\alpha(0<\alpha<1)$ in Algorithm 1 is the threshold for determining the minimum weight $w$, and it is set at 0.5 in this research.

Step 3: All combinations of vertices in clusters $C E$ are added to the context feature set $C F$. For example, in case of $C E_{1}=\left\{N F_{2}, N F_{3}\right\}$, three context features: $\left\{N F_{1}, N F_{2}\right\},\left\{N F_{1}, N F_{3}\right\},\left\{N F_{1}, N F_{2}, N F_{3}\right\}$ are newly added to $C F$.

Step 4: We modify Algorithm 1 (Column 1,6) by sorting the vertices in order, starting from the segments with the least number. Steps 2 to 4 are repeated again based on the modified Algorithm 1.

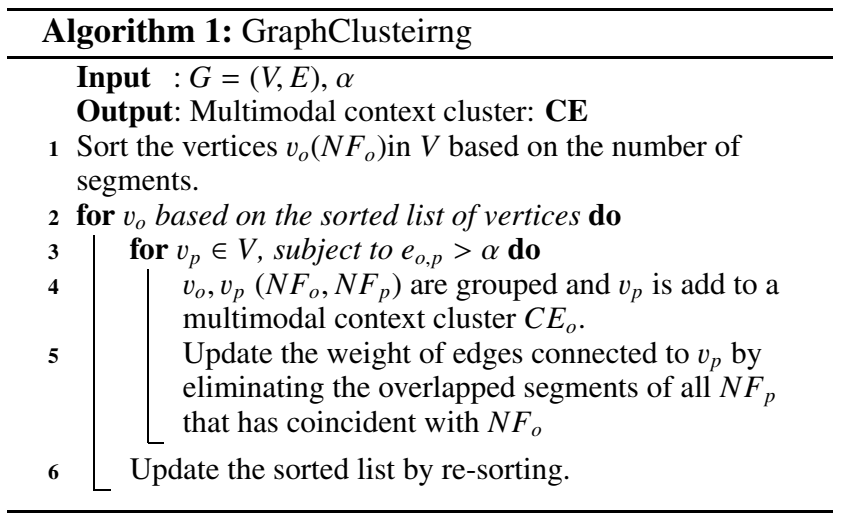

To extract many pattern candidates, we enhanced the original algorithm[26] by adding Steps 3 and 4, which are original processes. Step 3 aims to extract various kind of co-occurrence patterns such as even patterns, in which there is a temporal overlap between at least twodimensional segments. Step 4 tries to capture patterns that co-occur from low-occurrence patterns (rare patterns). Cooccurrence clustering results in extracting 195 multimodal patterns. Context feature $C F$ is calculated by counting the patterns observed in each episode.

\section{Multimodal Analysis and Modeling}

\subsection{Correlation Analysis}

We calculate the Pearson product-moment correlation coefficient between multimodal features (spoken word features, nonverbal features, context features) and questionnaire variables to measure storytelling performance. The objective of this pairwise correlation analysis is to calculate the linear correlation constant between each feature (variable) and the performance variable, independently. However, although the feature set exhibits significant linear correlation with the performance, it is not always the most effective feature set to predict it; the correlation value can be used as a criterion for feature selection [27] in machine learning. This analysis is also important in order to understand the relationship between each set of variables, and it was widely used in many researches for this purpose [12], [22] to understand it. These results of the pairwise correlation are also important as a reference for a future work to develop a model to predict other kinds of performance (explanation in different setting).

Table 3 shows the results of correlation analysis for verbal and nonverbal features (VF and NVF in Table 2). We list the variables, which show significant correlation with more

Table 3 Significant correlation between storytelling performance and multimodal features $(p<0.05: *, p<0.01: * *)$

\begin{tabular}{|c|c|c|c|}
\hline \multirow{2}{*}{$\begin{array}{l}\text { Multimodal } \\
\text { cue }\end{array}$} & \multicolumn{2}{|c|}{ Best significance } & \multirow{2}{*}{$\begin{array}{l}\text { Performance } \\
\text { variables } \\
\text { with } p<0.05\end{array}$} \\
\hline & $\begin{array}{l}\text { Performance } \\
\text { variable }\end{array}$ & $\mathrm{r}$ & \\
\hline$E P L$ & $E_{8}$ (Live.) & $+0.41^{* *}$ & $E_{1-10}$ \\
\hline \multicolumn{4}{|c|}{$\begin{array}{l}\text { Speech Cues } \\
\end{array}$} \\
\hline$E S L$ & $E_{1}$ (Eloquent) & $+0.27^{*}$ & $E_{1,3,4,8}$ \\
\hline$L S T$ & $E_{1}$ (Eloquent $)$ & $-0.27^{*}$ & $E_{1}$ \\
\hline$E S E_{\min }$ & $E_{10}(Q \& A)$ & $-0.49^{* *}$ & $E_{1-10}$ \\
\hline$E S E_{s t d}$ & $E_{10}(Q \& A)$ & $-0.28^{*}$ & $E_{1}$ \\
\hline$E S P_{\min }$ & $E_{6}$ (Compact.) & $-0.30^{*}$ & $E_{3-10}$ \\
\hline$E S P_{\text {mean }}$ & $E_{1}($ Eloquent $)$ & $-0.30^{*}$ & $E_{1-4,6,7}$ \\
\hline$E S P_{s t d}$ & $E_{5}$ (Precise.) & $+0.33^{* *}$ & $E_{2,4-7,9,10}$ \\
\hline \multicolumn{4}{|c|}{ Gaze Cues } \\
\hline$E G F$ & $E_{5}$ (Precise.) & $-0.27^{*}$ & $E_{1,5,7}$ \\
\hline$E M G$ & $E_{1}$ (Eloquent) & $-0.35^{* *}$ & $E_{1-10}$ \\
\hline$E G T$ & $E_{9}$ (Cooperate) & $+0.45^{* *}$ & $E_{1-10}$ \\
\hline \multicolumn{4}{|c|}{ Spoken Words } \\
\hline$E W\left(w_{6}\right)$ & $E_{8}$ (Live.) & $-0.41^{* *}$ & $E_{2-10}$ \\
\hline$E W\left(w_{7}\right)$ & $E_{1}$ (Eloquent) & $-0.49^{* *}$ & $E_{1-10}$ \\
\hline$E W\left(w_{8}\right)$ & $E_{1}$ (Eloquent) & $+0.53^{* *}$ & $E_{1-10}$ \\
\hline$E W\left(w_{9}\right)$ & $E_{10}(Q \& A)$ & $-0.40^{* *}$ & $E_{1-10}$ \\
\hline$E W\left(w_{10}\right)$ & $E_{9}$ (Cooperate) & $+0.27^{*}$ & $E_{5,7,9}$ \\
\hline$L W\left(w_{3}\right)$ & $E_{8}$ (Live.) & $+0.30^{*}$ & $E_{2,3,7,8}$ \\
\hline$L W\left(w_{4}\right)$ & $E_{1}$ (Eloquent) & $+0.31^{* *}$ & $E_{8,10}$ \\
\hline$L W\left(w_{7}\right)$ & $E_{2}$ (Enthus) & $-0.46^{* *}$ & $E_{1-10}$ \\
\hline$L W\left(w_{8}\right)$ & $E_{2}$ (Enthus) & $+0.32^{* *}$ & $E_{1-4,6,7,9,10}$ \\
\hline$L W\left(w_{9}\right)$ & $E_{2}$ (Enthus) & $+0.38^{* *}$ & $E_{1-10}$ \\
\hline
\end{tabular}


than two indexes of the storytelling performance in Table 3 . Correlations with $p<0.05$ are denoted by $*$ and $p<0.01$ by **. $r$ denotes the correlation index which shows the highest significance. The performance variables in Table 3 denote the most significant variable in $E_{1-10}$. The right-most row in Table 3 includes the other performance variables which exhibit significant correlation $(p<0.05)$.

\subsubsection{Verbal Cues and Storytelling Performance}

From Table 3, the frequencies of $w_{6}$ (particle), used by storytellers, and $w_{7}$ (auxiliary verb), by storytellers and listener, show significant negative correlation with $E_{2-10}$ and $E_{1-10}$, respectively. This indicates that lower scores tend to keep to episodes in which particles and auxiliary verbs are often used. The frequency of $w_{8}$ (interjection and backchannel) used by storytellers and listener exhibits significant positive correlation with almost all variables. Interjections and backchannel are well known as important cues of listener behavior. This means that higher scores occur with episodes in which interjections and backchannel are used often.

The frequency of $w_{9}$ (filler) of storytellers shows a maximum negative correlation $(-0.40)$ with $E_{10}(Q \mathcal{F} A)$. Conversely, the frequency of $w_{9}$ (filler) of the listener has significant positive correlation with $E_{1-10}$. This is assuming that fillers are often used by the storyteller in episodes with lower scores, and by the listener in those with higher scores.

Regarding the frequency of $w_{3,4}$, verb and adjective of the listener $L W\left(w_{3,4}\right)$ is significantly positively correlated with some variables. The frequency of $w_{10}$ (laugh of storytellers) $E W\left(w_{10}\right)$ shows significant positive correlation with some variables. It is natural that laughter is an important feature linked to lively conversation.

\subsubsection{Nonverbal Cues and Storytelling Performance}

From Table 3, we see that the time length of an episode $E P L$ shows significant positive correlation with all storytelling performance values $E_{1-10}$. This is expected, because storytellers need sufficient time to lay out the details of the story.

Regarding speech cues, the speech length of storytellers $(E S L)$ shows significant positive correlation with $E_{1,3,4,8}$. It shows the maximum correlation (0.27) with $E_{1}$ (Eloquent). Conversely, the count of utterance of the listener ( $L S T)$ shows maximum negative correlations $(-0.27)$ with $E_{1}$. This indicates that lower scores of eloquence tend to occur when the listener talks at great length. Prosodic features also exhibit significant correlations. In particular, the minimum energy in speech of storytellers has a negative correlation $(-0.49)$ with $E_{10}((Q \& A))$. The standard deviation of the pitch shows a positive correlation $(0.33)$ with $E_{5}$ (Precise.).

Regarding gaze cues, the time length during which attention is focused on the storytellers $(E G F)$ shows a significant negative correlation with $E_{1,5,7}$. It shows a maximum correlation $(-0.27)$ with $E_{5}$ (Precise.). The time length of
Table 4 Top 5 of significant correlation between storytelling performance and context features $(p<0.05: *, p<0.01: * *)$

\begin{tabular}{|l|l|l|c|}
\hline & \multirow{2}{*}{$\begin{array}{l}\text { Interpretation } \\
\text { of co-occurrent patterns }\end{array}$} & \multicolumn{2}{|c|}{ Best significance } \\
\cline { 3 - 4 } & & $\begin{array}{l}\text { Performance } \\
\text { variable }\end{array}$ & $\mathrm{r}$ \\
\hline$C E_{1}$ & $P_{E 1}$ speaks with looking at $P_{E 2}$ & $E_{5}$ Precise. & $+0.32^{* *}$ \\
\hline$C E_{2}$ & $\begin{array}{l}P_{L} \text { nods and } P_{E 2} \text { looks at } P_{E 1} \\
\text { while } P_{E 1} \text { speaks }\end{array}$ & $E_{5}$ Precise. & $+0.32^{* *}$ \\
\hline$C E_{3}$ & $\begin{array}{l}P_{E 2} \text { nods and looks at } P_{E 1} \\
\text { while } P_{E 1} \text { speaks with hand gesture }\end{array}$ & $E_{2}$ (Enthus) & $+0.31^{*}$ \\
\hline$C E_{4}$ & $\begin{array}{l}P_{E 2}\left(P_{L}\right) \text { looks at } P_{E 1}\left(P_{E 2}\right) \\
\text { while } P_{E 1} \text { speaks }\end{array}$ & $E_{1}$ (Eloquent) & $+0.31^{*}$ \\
\hline$C E_{5}$ & $\begin{array}{l}P_{L} \text { nods while } P_{E 1} \\
\text { speaks with hand gesture }\end{array}$ & $E_{2}$ (Enthus) & $+0.28^{*}$ \\
\hline
\end{tabular}

mutual gaze between the storytellers $(E M G)$ also shows a significant negative correlation with all variables and a maximum correlation $(-0.35)$ with $E_{1}$ (Eloquent). This indicates that the storytellers gaze often at one another, rather than at the listener, in episodes with lower score. Many storytellers tend to talk to the participant who also knows the video content, when they lack confidence in their memories. The frequency of gaze transitions of the storytellers (EGT) shows a significant positive correlation with all variables and a maximum correlation (0.45) with $E_{9}$ (Cooperate). Significant correlations are not found between head and hand gesture cues.

\subsubsection{Context Features and Storytelling Performance}

Table 4 shows the context features (CF in Table 2) with significant correlations. Overall, 23 out of the 195 features discovered from clustering show significant correlations. In Table 2, we report only the results with the five highest correlation coefficient $r$ values, due to the space limitation. To visualize these results, we list an interpretation of the respective context features in the second row of Table 4 .

$C E_{1-4}$ are context features, which include a storyteller speaking. From $C E_{2}$, the context, in which the listener's head gesture $H E_{L}$ is observed while a storyteller is speaking, has a significant positive correlation with some storytelling variables. This indicates that the storyteller-listener interaction is an important cue linked to storytelling performance. $C E_{3}$, the context cues in which a storyteller speaks with hand gestures ( $S P_{E 1}$ co-occurs with $\left.H G_{E 1}\right)$, also exhibit significant positive correlations with storytelling performance variables. $C E_{5}$, the context cues in which a listener speaks with hand gestures $\left(S P_{L}\right.$ co-occurs with $\left.H G_{L}\right)$, exhibits a significant positive correlation with $E_{2}$ (Enthus). This context shows that a listener asks a storyteller questions using gestures. The above results show that using cooccurrence clustering can help us to discover context cues regarding the conversation structure, which are significantly correlated with it. Therefore, it shows the effectiveness of context feature extraction using co-occurrence clustering.

\subsection{Performance Prediction Based on a Regression Task}

Multivariate regression models are built using a ridge regression model with an L2-norm regularization term. We use a cross validation method to test the regression accu- 


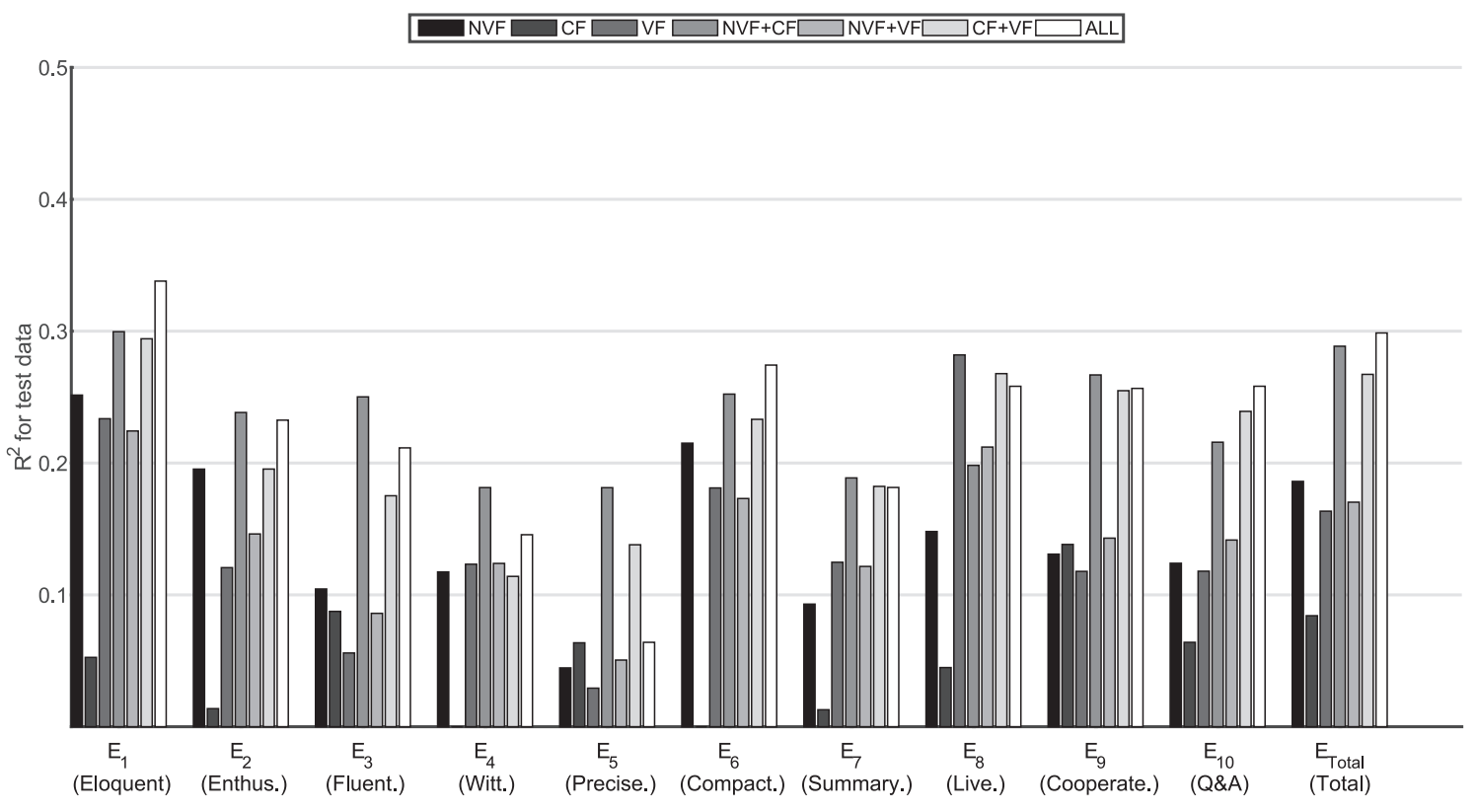

Fig. 4 Prediction accuracy in regression task

racy. The testing is conducted by omitting one episode from the 67 episodes. To address the first hypothesis (H1) and analyze the regression accuracy with each type of feature set, we prepare seven feature sets by combining verbal and nonverbal features.

- $N V F$ : nonverbal feature set including $S P, G, H G, H E$

- $C F$ : context feature set

- $V F$ : verbal feature set including $W$

- $N V F+C F$ : nonverbal feature set and context feature set

- $N V F+V F$ : verbal and nonverbal feature set

- $C F+V F$ : verbal and context feature set

- All: All feature set $(N V F+C F+V F)$

The EPL feature (time length of each episode) is included in all feature sets. We use PCA for $C F$, multimodal cooccurrence and the interaction feature set to reduce the dimensions. We use the cumulative energy ratio to determine the number of features, setting the cumulative energy ratio at 0.99 . After the process, the number of dimensions is determined at 30 from 195 . The storytelling performance $E_{1}-E_{10}$ and total performance $E_{\text {Total }}$, summed as $E_{1}-E_{10}$, are modeled as dependent variables. We calculate $R^{2}$ of the test data after training the model with each feature set using the ridge regression model. The weighting parameter of the regularization term influences the prediction performance in the ridge regression model. We choose the optimal parameter in $[20-150]$ through cross validation in the training data set. If the predicted value is larger than 10 or less than 1 (10 (1) is the maximum (minimum) value in a ten-point scale), the value is set to 10 or 1 automatically.

Figure 4 shows the $R^{2}$ value of the test data for each model built from each feature set. Comparing the results obtained from the models with verbal $V F$, nonverbal $N V F$, and nonverbal context features $C F$; the model with the verbal features gives the best results for $E_{4}$ : (Wittiness), $E_{7}$ :
(Summarization), $E_{8}$ : (Liveliness); the model with the nonverbal features for $E_{1}$ : (Eloquence), $E_{2}$ : (Enthusiasm), $E_{3}$ : (Fluentness), $E_{6}$ : (Compactness), $E_{10}:(Q \mathcal{E} A), E_{\text {Total }}$; and the model with nonverbal context features gives the best results for $E_{5}$ : (Preciseness), $E_{9}$ : (Cooperation). Wittiness $E_{4}$ and performance of summarization $E_{7}$ depend on the vocabulary of the storyteller, and it is reasonable that these variables are predicted well using verbal features. Eloquence, enthusiasm, fluentness, and $Q \& A$ are predicted well using nonverbal behavior rather than verbal features. Performance of cooperation is predicted best using context features. This means that the context features capture the performance of cooperation. On the other hand, the prediction accuracy for $E_{5}$ : (Preciseness) is lower than 0.1 in all the feature sets and we do not find an effective feature set to predict it.

This result shows that nonverbal features are effective in predicting performance, as compared to the individual feature sets. Comparing the results obtained from all the models, the model with $V F$ gives the best results for $E_{8}$, the model with $N V F+C F$ the best results for $E_{2,3-5,7,9}$, and the model with $A l l$ the best results for $E_{1,6,10, \text { Total }}$. Therefore, combining multimodal features is effective in predicting performance, in general. Furthermore, context feature $C F$ is more effective than nonverbal features in the regression task. In total, the model with $A l l$ gives the most accurate prediction $(0.338)$ for $E_{1}$ (Eloquent). These results show that a combination of nonverbal features $N V F+C F$ is effective to predict these variables.

\section{Analysis of Effective Feature Set for Prediction}

In order to address the second and the third hypotheses $(\mathrm{H} 2$, $\mathrm{H} 3$ ), we perform additional experiments and analyze the effective feature set for the prediction task. To analyze the contributions of the listener's verbal and nonverbal features, we develop new feature sets by removing the listener's fea- 
Table 5 Comparison of prediction accuracy $\left(R^{2}\right)$ between features extracted from speakers and all participants (speaker and listener)

\begin{tabular}{|c|c|c|c|c|c|c|c|c|c|c|c|c|c|c|}
\hline & \multicolumn{2}{|c|}{$N V F$} & \multicolumn{2}{|c|}{$C F$} & \multicolumn{2}{|c|}{$V F$} & \multicolumn{2}{|c|}{$N V F+C F$} & \multicolumn{2}{|c|}{$N V F+V F$} & \multicolumn{2}{|c|}{$C F+V F$} & \multicolumn{2}{|c|}{ All } \\
\hline & S peaker & Both & S peaker & Both & S peaker & Both & S peaker & Both & S peaker & Both & S peaker & Both & S peaker & Both \\
\hline$E_{1}$ & 0.216 & 0.252 & -0.056 & 0.053 & 0.224 & 0.234 & 0.240 & 0.300 & 0.158 & 0.224 & 0.241 & 0.294 & 0.226 & $\underline{0.338}$ \\
\hline$E_{2}$ & 0.195 & 0.195 & -0.068 & 0.014 & 0.110 & 0.121 & 0.161 & $\underline{0.238}$ & 0.137 & 0.146 & 0.193 & 0.195 & 0.097 & $\overline{0.233}$ \\
\hline$E_{3}$ & 0.088 & 0.104 & 0.104 & 0.087 & -0.003 & 0.056 & 0.117 & $\overline{\mathbf{0 . 2 5 0}}$ & 0.138 & 0.086 & 0.059 & 0.175 & 0.096 & 0.211 \\
\hline$E_{4}$ & 0.123 & 0.117 & -0.008 & -0.035 & 0.151 & 0.123 & 0.160 & $\overline{0.181}$ & 0.134 & 0.124 & 0.151 & 0.114 & 0.134 & 0.146 \\
\hline$E_{5}$ & 0.050 & 0.045 & 0.014 & 0.064 & 0.004 & 0.029 & 0.076 & $\overline{0.181}$ & 0.075 & 0.051 & 0.035 & 0.138 & 0.098 & 0.064 \\
\hline$E_{6}$ & 0.201 & 0.215 & -0.141 & -0.020 & 0.194 & 0.181 & 0.215 & $\overline{0.252}$ & 0.164 & 0.173 & 0.210 & 0.233 & 0.190 & 0.274 \\
\hline$E_{7}$ & 0.103 & 0.093 & -0.028 & 0.013 & 0.093 & 0.125 & 0.183 & 0.189 & 0.154 & 0.122 & 0.130 & 0.182 & 0.160 & $\overline{0.181}$ \\
\hline$E_{8}$ & 0.147 & 0.148 & -0.014 & 0.045 & 0.238 & 0.282 & 0.143 & $\overline{0.198}$ & 0.096 & 0.212 & 0.191 & 0.268 & 0.111 & 0.258 \\
\hline$E_{9}$ & 0.126 & 0.131 & 0.139 & 0.138 & 0.125 & $\overline{0.118}$ & 0.236 & 0.267 & 0.217 & 0.143 & 0.163 & 0.255 & 0.239 & 0.257 \\
\hline$E_{10}$ & 0.146 & 0.124 & 0.101 & 0.064 & 0.088 & 0.118 & 0.283 & $\overline{0.216}$ & 0.295 & 0.142 & 0.153 & 0.239 & 0.172 & 0.258 \\
\hline$E_{\text {Total }}$ & 0.173 & 0.186 & 0.006 & 0.084 & 0.132 & 0.163 & 0.224 & 0.289 & 0.186 & 0.170 & 0.182 & 0.267 & 0.201 & 0.299 \\
\hline Mean & 0.143 & 0.146 & 0.004 & 0.046 & 0.123 & 0.141 & 0.185 & 0.233 & 0.159 & 0.145 & 0.155 & 0.215 & 0.157 & 0.229 \\
\hline
\end{tabular}

tures from $N V F, C F$, and $V F$. We set the new verbal and nonverbal feature set for only speakers as $N V F_{S \text { peaker }}$ and $V F_{S \text { peaker }}$ by removing all the listener's features $L *$ (e.g., in Table 2) from the feature sets $N V F$ and $V F$. We also remove context features including the listener's nonverbal features (e.g., a listener $\left(P_{L}\right)$ looks at a storyteller $\left.\left(P_{E 1}\right)\right)$ from context feature set $C F$ and define the context feature set only for the speakers as $C F_{\text {Speaker }}$. We form seven feature sets in the same manner as in Sect. 6.2 by combining $N V F_{S \text { peaker }}$, $V F_{S \text { peaker }}$ and $C F_{\text {S peaker }}$. We also define $*_{\text {Both }}$ which denotes the feature set extracted from both the speaker and the listener.

We perform experiments on the regression task under the same settings as in Sect. 6.2. Table 5 shows the comparison of the prediction accuracy on the regression task between models trained with $*_{s \text { peaker }}$ and with $*_{\text {Both }}$. In Table 5, the value in the row of $S$ peaker, Both denotes the prediction accuracy $\left(R^{2}\right)$ obtained by model with $*_{S \text { peaker }}, *_{\text {Both }}$. Note that the values in rows of Both correspond to the values of the bar graph in Fig. 4. We compare the prediction performance of fourteen feature sets (seven from $S$ peaker and 7 from Both). Bold typed values denote the three best accuracies in the fourteen sets. The bold and underlined value denotes the best accuracy.

\subsection{Effectiveness of Listener's Features (H2)}

By comparing the accuracy obtained from the model with $*_{\text {S peaker }}$ to that with $*_{\text {Both }}$, we are able to analyze the contribution of the listener's features to the prediction task. From Table 5, the accuracy of the $S$ peaker is virtually the same as that of Both in $N V F, C F$, and $V F$. The accuracy of $S$ peaker is better than that of Both in $N V F+V F$. The tendency is clear in the prediction task of $E_{10}(Q \mathcal{E} A)$. These results show that features extracted from storytellers in $N V F+V F$ are effective, whereas the feature set extracted from the listener provides lower accuracy.

In contrast, the accuracy of Both is better than that of $S$ peaker in $N V F+C F, C F+V F$, and All. In particular, the listener's feature set in $C F+V F$ improves, from 0.05 to 0.17 , in the prediction task of $E_{3}$, and the set in All improves, from 0.09 to 0.23 , in the task of $E_{2}$. The mean accuracy in $E_{1-10 \text {,Total }}$ of $*_{\text {Both }}$ is better than that of $*_{\text {Speaker }}$ for all feature sets $(*)$ except $N V F+V F$. Consequently, these results suggest that the listener's feature set is effectively improves the prediction accuracy, when multimodal features are fused. Thus, $\mathrm{H} 2$ is supported.

\subsection{Effectiveness of Context Features (H3)}

We discuss whether the context features improve the regression accuracy. First, the model with $(N V F+C F)_{B o t h}$ gives the best accuracy in seven variables $\left(E_{2-5,7}\right.$ and $\left.E_{9}\right)$ and the best mean accuracy (Mean). Consequently, context features $(C F)$ are most effective in improving the accuracy by combining multimodal features. It also shows that combining nonverbal features $(N V F$ and $C F$ ) improve the accuracy more than fusing verbal features. However, the mean accuracy with $N V F_{\text {Both }}$ and $V F_{\text {Both }}$ is better than that with $C F_{B o t h}$, showing that $C F$ is not effective in improving the accuracy as a unimodal feature set. Therefore, $C F$ contributes to improve the accuracy when it is fused with $N V F_{\text {Both }}$ and $V F_{\text {Both }}$. The mean accuracy in $(N V F+C F)_{\text {Both }}$ $(0.23)$ is better than that in $N V F_{B o t h}(0.14)$. The mean accuracy in $(C F+V F)_{\text {Both }}(0.21)$ is better than that in $V F_{\text {Both }}$ (0.14). Thus the hypothesis H3 is supported.

\section{Discussion}

\subsection{Limitations of the Proposed Approach}

The technical limitations of the approach proposed in this study are summarized.

1. The proposed framework employs conversation data, which are segmented into episode units.

2. The proposed framework works well on the performanceprediction task when the role of each participant (storyteller or listener) is assigned, and the content of the story is common in the groups.

3. The situation where two storytellers collaboratively tell a story to a listener is somewhat special. The special case can be observed in business setting (e.g. more than two sales staff explain of product to a client) or in education setting (e.g. some students teach one student cooperatively in group work).

Although the proposed framework was not evaluated in 
setting with one-to-one interaction which is a generic case, the framework also works on the dyadic setting if the roles are fixed, because experimental results show that the single listener's features are effective.

4. Evaluation is conducted using manual annotation data. In order to apply this framework to an autonomous system, it must be evaluated using features extracted in an automated manner. In particular, verbal features are extracted from manual transcriptions; this needs to be replaced to a speech recognizer.

\subsection{Achieved Prediction Accuracy}

We discuss how the best accuracy $R^{2}=0.299$ for the total performance $E_{\text {Total }}$ is interpreted by comparing the accuracy with that obtained in previous research. As a reference, [18] reports that the regression accuracy for persuasiveness of applicants in a job interview was $R^{2}=0.118$ using a random forest model. Therefore, the best result here $\left(R^{2}=0.299\right)$ is comparatively good.

The binary prediction accuracy is another measure for evaluating regression accuracy, and it can be used to calculate whether the predicted performance is higher or lower than a threshold value (also used in [8], [12]). In the task, real performance values (1-10) are converted to binary values (high or low) using the median value as the threshold. The predicted performance value, which is output from the trained model $M_{\text {all }}$, is converted to a binary value in the same manner. Therefore, evaluating the accuracy of the regression model $M_{\text {all }}$ by binary classification accuracy of test data, the classification accuracy of $M_{\text {all }}$ is $74.5 \%$, which is higher than the $50.0 \%$ accuracy level of the binary classification task.

As a reference, [8] reports that binary prediction accuracy of a speaker's persuasiveness was $70.8 \%$ using multimodal features. However, we cannot compare our results $(74.5 \%)$ with those of [8] (70.8\%) directly, because [8] use speaker data only, based on a monologue, and not dialog data such as in the present study. Nevertheless, our results appear improved in comparison.

\subsection{Generic Explanation Performance Modeling}

The multimodal features and the present machine-learning approach are available for modeling the performance of a general type of explanation, which has similar characteristics with storytelling. This is because the extracted language features (the part of speech, laugh, and filler) are also available as simple and generic features for capturing the participants' attitude during a presentation or explanation. In addition, extracted nonverbal features including head direction approximated as gaze direction, head and hand gesture, and prosody are also available for capturing it.

However, it is not clear how much these features are effective for the prediction task in other situations, including presentations and explanations. For example, when a vendor conducts storytelling to a client, in a formal situation such as a business meeting, he has to assume a polite attitude, while the client is not always cooperative with vendors. We expect that values of multimodal features in such formal settings may be different from those in settings that are more casual. A future work would be to apply this framework for prediction tasks in other kinds of situations (presentation and explanation) and compare the effectiveness of each feature in the prediction task of each performance. We believe that our multimodal modeling framework is a fundamental and useful tool that enables us to analyze the performance in various kinds of situations.

\subsection{Improving Prediction Accuracy}

Experimental results show that the model combining nonverbal features $N V F+C F$ provides the best accuracy in six out of the ten investigated kinds of performances. Thus, different nonverbal features and their combinations should be examined, in order to improve the performance prediction accuracy even further. Regarding prosodic features, a next step is to distinguish utterance spoken with a louder voice than usual to that spoken slowly. Moreover, regarding gaze features, more kinds of states should be distinguished for visual focus of attention, e.g., whether the listener (storyteller) is looking at the storyteller's (listener's) face or gesture. Computational gaze analysis techniques [28] are useful for this purpose. For features of hand and head gesture, a next step is to determine the category of hand gesture [23] and extract the amount of head movement.

\section{Conclusion}

We presented a multimodal analysis to infer the storytelling performance. We proposed group nonverbal features extracted from verbal, nonverbal, and context features extracted using time-series co-occurrence clustering based on three hypotheses. The correlation analysis indicated that context features such as gaze state transition and storyteller's speech accompanying hand gestures are strongly correlated. Furthermore, regression modeling showed that combinations of nonverbal and context features improve the performance prediction accuracy. We show that the listener's features and context features are effective to predict it, through an additional experiment using feature sets extracted from storytellers (Speaker). These results suggest that three hypotheses are important to improve the prediction accuracy. An important future direction of this work is to apply this model for other kinds of storytelling conversation (Sect. 8.3). In addition, we should extract various kinds of nonverbal features to improve the accuracy (Sect. 8.4).

\section{Acknowledgments}

We would like to thank both reviewers for their insightful comments. We also thank the support of JSPS KAKENHI (25730132, 15K00300, 25280076). 


\section{References}

[1] J.S. Brown, Storytelling in organizations: Why storytelling is transforming 21 st century organizations and management, Routledge, 2005.

[2] D.H. Andrews, T.D. Hull, and J.A. Donahue, "Storytelling as an instructional method: Definitions and research questions," Interdisciplinary Journal of Problem-based Learning, vol.3, no.2, p.3, 2009.

[3] A. Tabensky, "Non-verbal resources and storytelling in second language classroom interaction," Journal of Applied Linguistics, vol.5, no.3, pp.321-348, 2008.

[4] J.K. Burgoon, T. Birk, and M. Pfau, "Nonverbal behaviors, persuasion, and credibility," Human Communication Research, vol.17, no.1, pp.140-169, 1990.

[5] E. Strangert and J. Gustafson, "What makes a good speaker? subject ratings, acoustic measurements and perceptual evaluations," INTERSPEECH, pp.1688-1691, 2008.

[6] D. McNeill, Gesture and Thought, University of Chicago Press, 2008.

[7] S.R.H. Langton, R.J. Watt, and V. Bruce, "Do the eyes have it? cues to the direction of social attention," Trends in cognitive sciences, vol.4, no.2, pp.50-59, 2000.

[8] S. Park, H.S. Shim, M. Chatterjee, K. Sagae, and L.-P. Morency, "Computational analysis of persuasiveness in social multimedia: A novel dataset and multimodal prediction approach," Proc. of ACM ICMI, pp.50-57, 2014.

[9] J.B. Bavelas, L. Coates, and T. Johnson, "Listeners as co-narrators.," Journal of personality and social psychology, vol.79, no.6, pp.941-952, 2000.

[10] K. Otsuka, "Multimodal conversation scene analysis for understanding people's communicative behaviors in face-to-face meetings," in Human Interface and the Management of Information. Interacting with Information, vol.6772, pp.171-179, Springer, 2011.

[11] F. Pianesi, N. Mana, A. Cappelletti, B. Lepri, and M. Zancanaro, "Multimodal recognition of personality traits in social interactions," Proc. of ICMI, New York, NY, USA, pp.53-60, 2008.

[12] O. Aran and D. Gatica-Perez, "One of a kind: Inferring personality impressions in meetings," Proc. of ICMI, pp.11-18, 2013.

[13] C.-Y. Weng, W.-T. Chu, and J.-L. Wu, "Rolenet: Movie analysis from the perspective of social networks," IEEE Trans. on Multimedia, vol.11, no.2, pp.256-271, 2009.

[14] A. Vinciarelli, "Speakers role recognition in multiparty audio recordings using social network analysis and duration distribution modeling," IEEE Trans. on Multimedia, vol.9, no.6, pp.1215-1226, 2007.

[15] N.P. Garg, S. Favre, H. Salamin, D.H. Tür, and A. Vinciarelli, "Role recognition for meeting participants: An approach based on lexical information and social network analysis," Proc. of ACM Multimedia, pp.693-696, 2008.

[16] D. Sanchez-Cortes, O. Aran, M.S. Mast, and D. Gatica-Perez, "A nonverbal behavior approach to identify emergent leaders in small groups," IEEE Trans. on Multimedia, vol.14, no.3, pp.816-832, 2012.

[17] D. Gatica-Perez, I. McCowan, D. Zhang, and S. Bengio, "Detecting group interest-level in meetings," Proc. of IEEE ICASSP, pp.489-492, 2005.

[18] L.S. Nguyen, D. Frauendorfer, M.S. Mast, and D. Gatica-Perez, "Hire me: Computational inference of hirability in employment interviews based on nonverbal behavior," IEEE Trans. on Multimedia, vol.16, no.4, pp.1018-1031, June 2014.

[19] L. Batrinca, G. Stratou, A. Shapiro, L.-P. Morency, and S. Scherer, "Cicero-towards a multimodal virtual audience platform for public speaking training," Intelligent Virtual Agents, vol.8108, pp.116-128, Springer, 2013.

[20] L.-P. Morency, I. de Kok, and J. Gratch, "Context-based recognition during human interactions: Automatic feature selection and encod- ing dictionary," Proc. of ACM ICMI, pp.181-188, 2008.

[21] D. Jayagopi and D. Gatica-Perez, "Mining group nonverbal conversational patterns using probabilistic topic models," Trans. Multi., vol.12, no.8, pp.790-802, 2010.

[22] D. Jayagopi, D. Sanchez-Cortes, K. Otsuka, J. Yamato, and D. Gatica-Perez, "Linking speaking and looking behavior patterns with group composition, perception, and performance," Proc. of ACM ICMI, pp.433-440, 2012.

[23] S. Okada, M. Bono, K. Takanashi, Y. Sumi, and K. Nitta, "Contextbased conversational hand gesture classification in narrative interaction," Proc. of ACM ICMI, pp.303-310, 2013.

[24] D. McNeill, Hand and Mind: What Gestures Reveal about Thought, Psychology/cognitive science, University of Chicago Press, 1996.

[25] L.S. Kennedy and D.P. Ellis, "Laughter detection in meetings," NIST ICASSP 2004 Meeting Recognition Workshop, pp.118-121, National Institute of Standards and Technology, 2004.

[26] A. Vahdatpour, N. Amini, and M. Sarrafzadeh, "Toward unsupervised activity discovery using multi-dimensional motif detection in time series," Proc. IJCAI, pp.1261-1266, 2009.

[27] A.L. Blum and P. Langley, "Selection of relevant features and examples in machine learning," Artificial intelligence, vol.97, no.1-2, pp.245-271, 1997.

[28] A. Kimura, R. Yonetani, and T. Hirayama, "Computational models of human visual attention and their implementations: A survey," IEICE Transactions, vol.96-D, no.3, pp.562-578, 2013.

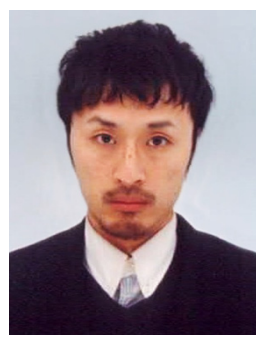

Shogo Okada received Ph.D. degree in Computer Science from the Tokyo Institute of Technology 2008. He worked in Kyoto University to 2011 as a project assistant professor. Currently, he is an assistant professor of School of Computing, Tokyo Institute of Technology. His main research interests are human behavior analysis and social signal processing. He is a member of, Association for Computing Machinery (ACM), Japanese Society for Artificial Intelligence (JSAI).

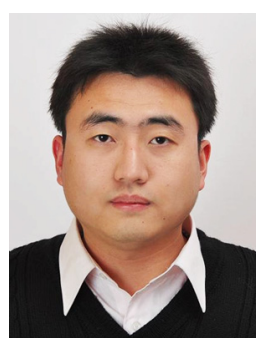

Mi Hang received M.E. degree in Computer Science from the Tokyo Institute of Technology, in 2014. His main research is human behavior analysis.

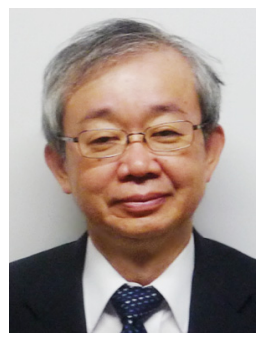

Katsumi Nitta received his B.E., M.E. and Dr.Eng. from Tokyo Institute of Technoloy in 1975, 1977 and 1980, respectively. In 1980, he joined Electrotechnical Laboratory as a computer scientist, and in 1989, he joined Institute for New Generation Computer Technology. Since 1996. he has been a Professor of the Interdisciplinary Graduate School of Science and Engineering, Tokyo Institute of Technology. His research interest includes the legal informatics and man-machine interaction. He is a member of the Information Processing Society of Japan (IPSJ), the Japanese Society for Artificial Intelligence (JSAI). 